\title{
The Underestimated Problem of Intra-Oral Halitosis in Dental Practice: An Expert Consensus Review
}

This article was published in the following Dove Press journal:

Clinical, Cosmetic and Investigational Dentistry

\author{
Stefan Renvert (iD ${ }^{1-3}$ \\ Michael J Noack (iD) 4 \\ Christophe Lequart ${ }^{5}$ \\ Silvia Roldán ${ }^{6}$ \\ Marja L Laine ${ }^{7}$
}

'Department of Oral Health, Kristianstad University, Kristianstad, Sweden; ${ }^{2}$ School of Dental Science, Trinity College, Dublin, Ireland; ${ }^{3}$ Blekinge Institute of Technology, Karlskrona, Sweden; ${ }^{4}$ Department of Operative Dentistry and Periodontology, University of Cologne, Cologne, Germany; ${ }^{5}$ Private practice, Sorigny, Union Française pour la Santé BuccoDentaire (UFSBD), Paris, France; ${ }^{6}$ ETEP (Etiology and Therapy of Periodontal Diseases) Research Group, University Complutense, Madrid, Spain;

${ }^{7}$ Department of Periodontology, Academic Center for Dentistry Amsterdam (ACTA), University of Amsterdam and Vrije Universiteit Amsterdam, Amsterdam, the Netherlands
Correspondence: Stefan Renvert Department of Oral Health, Faculty of Health Sciences, Kristianstad University, SE-29I 88 Kristianstad, Sweden

Tel +460442503000

Email stefan.renvert@hkr.se

\begin{abstract}
Approximately $90 \%$ of halitosis cases originate within the oral cavity (intra-oral halitosis). With a focus on intra-oral halitosis, this narrative review article provides a current summary of the epidemiology, diagnosis and management of halitosis and discusses practical considerations for healthcare professionals (HCPs), including dentists, dental hygienists, general practitioners, community pharmacists, nurses and medical specialists. MEDLINE and PubMed were searched up to 31 December 2019. Additional information was sourced from reference lists of relevant published literature. Full-text articles which reported on the epidemiology, diagnosis and management of halitosis were considered for inclusion. Halitosis affects substantial numbers of individuals globally and is an underestimated problem in dental practice. Current estimates of the prevalence of halitosis, in addition to diagnostic methods and management considerations for halitosis, are discussed. Although not a life-threatening condition, halitosis has a significant impact on patients' quality of life and can result in psychological consequences including social, professional and affective limitations. Using a simple step-wise approach for diagnosis and treatment, dentists and dental hygienists are ideally placed to respond to an initial consultation for halitosis.
\end{abstract}

Keywords: bad breath, halitosis, intra-oral halitosis, oral health

\section{Introduction}

The term halitosis is derived from the merger of the Latin halitus (breath) and Greek osis (pathological process) to describe a condition that refers to an unpleasant odor from the mouth or "bad breath". Halitosis is sometimes called chronic bad breath, bad breath, oral malodor, or various other terms such as tongue malodor, fetor ex ore, and fetor oris. ${ }^{1-7}$ However, bad breath is a general term which usually has negative connotations and is associated with stigma possibly due to the word 'bad'. Halitosis and bad breath are most likely simply different ways of naming the same symptom (halitosis may be a condition or a symptom of underlying disease); in simple terms, halitosis can be defined as a socially unacceptable level of breath odor. A preferred alternative term, which removes some of the stigma and taboo associated with the term 'bad breath', is 'breath odor'.

Halitosis is considered to be one of the most unattractive aspects of social interactions. ${ }^{8}$ Individuals with halitosis often experience psychological consequences that can lead to social, professional, and affective limitations, ${ }^{9,10}$ and poor oral health-related quality of life. ${ }^{11}$ Research has identified social anxiety 
disorder as the most common psychopathology associated with halitosis complaints. ${ }^{10,12,13}$

This narrative article is based on current literature reviewed and discussed among experts in the field of halitosis and periodontology at an Advisory Board meeting about the current status of halitosis. In addition, MEDLINE and PubMed were searched up to 31 December 2019 (search terms included 'halitosis', 'epidemiology', 'diagnosis', 'treatment', 'clinical trial') and full-text articles which reported on the epidemiology, diagnosis and management of halitosis were considered for inclusion; information was also sourced from reference lists of relevant published literature. The objectives of the article are to: (1) improve general awareness of halitosis, with a particular focus on intra-oral halitosis; (2) build the relevance of halitosis in dental practice, and (3) provide practical guidance for healthcare professionals (HCPs) on how to best manage/treat halitosis.

\section{Classification and Causes of Halitosis}

Halitosis is classified as genuine halitosis (intra-oral and extraoral halitosis), transient (temporary) halitosis and pseudohalitosis/halitophobia (imaginary bad breath). When diagnosing/ treating patients with suspected halitosis, it is important to distinguish between the different types of halitosis.

In contrast to general perceptions, the oral cavity is responsible for approximately $90 \%$ of halitosis cases (intra-oral halitosis). ${ }^{14-16}$ Intra-oral halitosis is caused by oral diseases (eg gingivitis, periodontitis, pericoronitis, necrotizing ulcerative gingivitis/periodontitis, dental caries, oral ulceration and oral malignancy), tongue coating, food impaction, and dry socket. ${ }^{4,17,18}$

A Belgian study of 2000 subjects who attended a halitosis clinic found that intra-oral halitosis was due to tongue coating $(43 \%)$, gingivitis/periodontitis $(11 \%)$ or a combination of both (18\%). ${ }^{15}$ Other factors associated with intra-oral halitosis include xerostomia and hyposalivation (dry mouth), ${ }^{19,20}$ an imbalanced microbiome/accumulation of oral bacteria, open caries lesions, stress, and age-related factors such as decreased oral mobility, polymedication, or the use of removable prostheses (eg retentive areas and porous surfaces of prostheses, including under dental bridges). ${ }^{4,21}$

Volatile sulfur compounds (VSCs), such as hydrogen sulfide $\left(\mathrm{H}_{2} \mathrm{~S}\right)$ and methyl mercaptan $\left(\mathrm{CH}_{3} \mathrm{SH}\right)$ are primarily responsible for the odor associated with intra-oral halitosis. ${ }^{4,7}$ Dimethyl sulfide $\left(\left[\mathrm{CH}_{3}\right]_{2} \mathrm{~S}\right)$ is mainly associated with extra- oral halitosis cases. The formation of VSCs in the oral cavity results from the degradation of organic substrates by anaerobic bacteria and may be affected by saliva secretion, reduced oxygen concentration, and bacterial metabolism. ${ }^{7}$ Tongue coating is considered to be the main cause of intra-oral halitosis (Figure 1). ${ }^{22-25}$ However, although the tongue coating microflora is thought to play an essential role in intra-oral halitosis, the exact microbiological causative factors of intraoral halitosis remain unclear. A recent study demonstrated that the metabolic profiles of the tongue coating microbiomes of patients with intra-oral halitosis were significantly different to those of healthy controls. ${ }^{26}$ Branched-chain fatty acids (BCFAs), 3-fumaryl pyruvate, and acetyl phosphate, are potential key substances in intra-oral halitosis, with BCFAs, the predominant metabolite in intra-oral halitosis, shown to possibly underlie tongue coating formation. Moreover, acetyl phosphate has a clear association with the $\mathrm{H}_{2} \mathrm{~S}$-producing metabolic pathway and anaerobic metabolism. ${ }^{26}$ Evidence for the possible role of Candida species in intra-oral halitosis is conflicting, with some authors reporting a positive correlation between VSC measurements and Candida culture test results, ${ }^{27}$ whereas others have reported no relationship between the presence of Candida and oral halitosis. ${ }^{28}$

Extra-oral halitosis, estimated to be responsible for approximately $5-10 \%$ of all halitosis cases, can result from numerous causes including ear/nose/throat (ENT) or respiratory diseases (eg sinusitis, tonsillitis, bronchiectasis, malignancy) and foreign bodies in the airway. $4,29,30$ Specific chronic diseases (eg gastric reflux, diabetes,

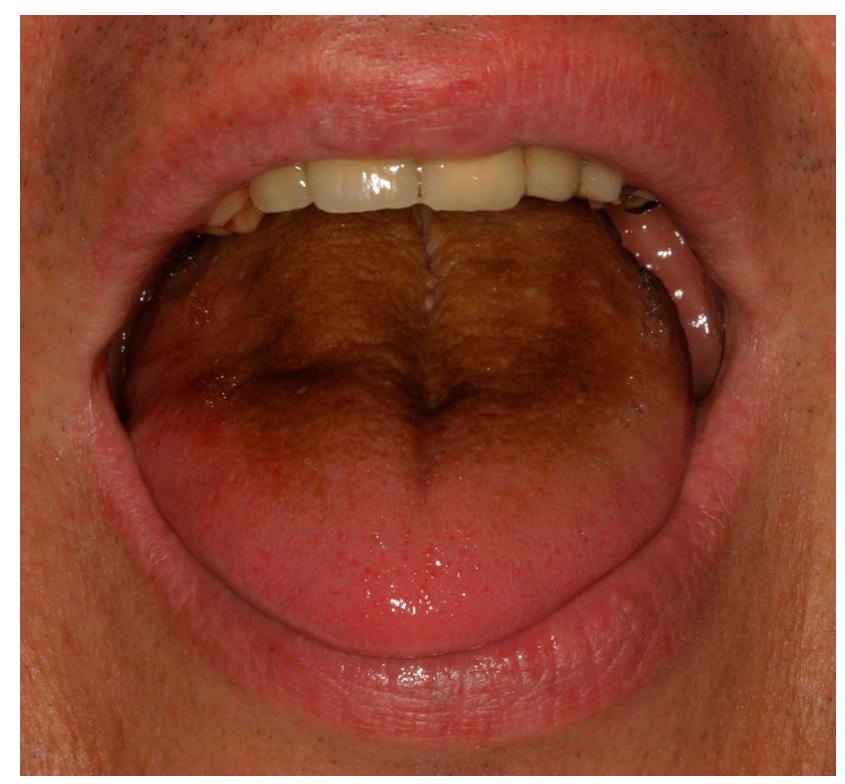

Figure I Tongue coating. 
carcinoma, and liver or kidney disease), drugs (eg paracetamol [acetaminophen], chloral hydrate, dimethyl sulfoxide, disulfiram, nitrate and nitrites, phenothiazines, and amphetamines) may also result in extra-oral halitosis. ${ }^{4,29,31}$

More recently, a revised etiologic classification system has been proposed for halitosis which divides pathologic halitosis into Type 0 (physiologic), Type 1 (oral), Type 2 (airway), Type 3 (gastroesophageal), Type 4 (blood-borne), and Type 5 (subjective); the subjective category is further divided into psychologic and neurogenic causes. ${ }^{32}$ This new system allows for multiple diagnoses in the same patient, reflecting the multifactorial nature of halitosis.

\section{Epidemiology}

There are currently no robust data regarding the prevalence of halitosis. ${ }^{33}$ Although many studies report the estimated prevalence of halitosis, the majority do not provide a clear definition of the type of halitosis. However, estimates reported in the published literature indicate that the general prevalence of halitosis is approximately $30 \%$. A recent systematic review and meta-analysis reported an estimated halitosis prevalence of $31.8 \%$, with high heterogeneity between studies. ${ }^{34}$ Almost all $(89.3 \%)$ of an online panel of $>1000$ individuals, who were considered to be representative of the population of The Netherlands, reported regularly encountering people with halitosis $\left(40 \%\right.$ at least once per week). ${ }^{8}$ One review of the epidemiology of halitosis reported a wide range for its prevalence, ranging from $2.4 \%$ for severe halitosis to $42 \%$ for subjectively reported halitosis. ${ }^{35}$ Moreover, a study from Pakistan reported a prevalence of $75 \%,{ }^{36}$ and there are also some recent data from Asia reporting high prevalence rates (44.9\% [Japan] and $65.9 \%$ [China]). ${ }^{37,38}$ The incidence of halitosis increases with age $^{39}$ and there appears to be a worldwide trend towards an increasing prevalence of halitosis in adults. ${ }^{34}$ A study of 124 individuals living in nursing homes in Sweden reported that halitosis occurred in over $50 \%$ of residents and was associated with the presence of hyposalivation, periodontal diseases, calculus, fixed prosthodontics and dementia. ${ }^{40}$

Epidemiology data in children are currently limited. Data from a cross-sectional study showed that halitosis was diagnosed in $17.3 \%$ of 150 children/adolescents (aged 6-12 years) who were scheduled for dental treatment at a pediatric dental clinic in Brazil; halitosis was significantly associated with a fair score on the Simplified Oral Hygiene Index, non-daily use of tooth floss, percentage of coated tongue, abnormal salivary flow, and dental caries. ${ }^{41}$ A smaller study, in 101 healthy children (median age 12 years), reported that halitosis was measured objectively in $37.6 \%$ of individuals, occurring more commonly in females, individuals with dental plaque on $>25 \%$ of the dental surfaces and children aged $>13$ years. ${ }^{42}$ A slightly lower prevalence of halitosis (23\%), based on an analysis of VSCs, was reported in a small study in 30 children (mean age 8.8 years). ${ }^{43}$

\section{Diagnostic Approach}

An accurate diagnosis is important in guiding management decisions in halitosis. ${ }^{44}$ A cross sectional study in 407 patients attending a breath odor consultation showed that, although all patients reported bad breath, only $72 \%$ had detectable breath malodor. ${ }^{14}$ Moreover, in the group without detectable breath malodor (presumed pseudohalitosis), more than three-quarters of patients had received prior diagnostics and treatments from other specialists, including approximately one-third who had received one or more gastroscopies and 14\% who had undergone ENT surgery.

The main diagnostic approaches for halitosis include: anamnesis (medical, dental and halitosis), assessment of oral health status (including soft tissue, dental and periodontal examination) and oral hygiene, tongue coating, and breath odor measurements. The patient's perception of their breath odor also needs to be evaluated. Although specialized halitosis clinics and individual centers have developed patient questionnaires, ${ }^{38,45,46}$ there appears to be an unmet need for the development of a simple, easy-to -use, internationally accepted and validated questionnaire.

Several methods have been used to assess the presence and extent of tongue coating; ${ }^{47-49}$ one of the simplest and well-accepted methods is the Winkel Tongue Coating Index (WTCI; Figure 2), ${ }^{4,49}$ or a modification of the WTCI (mWTCI). ${ }^{50}$ Lundgren and co-workers discovered that WTCI score 1 often seems to represent an increased keratinization of the papillae of the tongue rather than tongue coating. ${ }^{50}$ Since this may affect the validity of this index, they decided to delete score 1 . They concluded that the mWTCI was highly reproducible and related to the wet weight of tongue scrapings. The assessment of tongue coating can be used to indicate whether a patient is likely to have halitosis because of tongue coating (a high score on the WTCI does not necessarily mean that a patient has halitosis but it is an indicator that halitosis is more likely). As well as being a useful tool for dentists (as part of a regular check-up), the tongue coating index can also act as a motivating factor and educational tool for patients because they can see the amount of tongue coating. 


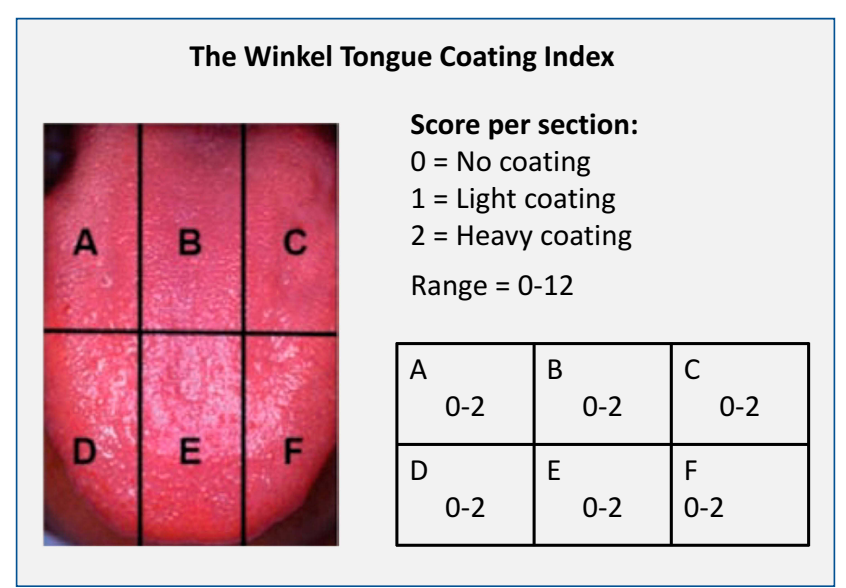

Figure 2 Winkel Tongue Coating Index. ${ }^{48,49}$ The dorsum of the tongue is divided into sections and the amount of tongue coating is graded subjectively (0-2) for each section. The total score, ranging from 0 to 12 , is calculated by adding the scores for each individual section.

Direct breath odor diagnostic methods include the organoleptic method, gas chromatography (eg, Oral Chroma $^{\mathrm{TM}}$ ), and/or portable sulfide monitoring. ${ }^{51}$

The organoleptic method is considered as the gold standard in the examination of breath malodor. It is easy to perform and most closely resembles the daily situation. In addition, the human nose can distinguish over 10,000 odors, much more than any device available in the market. ${ }^{52}$ To avoid any bias, it is preferred that this evaluation precedes all other evaluations. ${ }^{53,54}$ After closing their mouth for 1-3 minutes to concentrate the odor, the patient opens their mouth and breath odor can be evaluated either directly, with the clinician situated a short distance $(10 \mathrm{~cm})$ from the patient's mouth, or indirectly through a tube. The clinician grades the breath odor between 0 and 5: 0: no odor detectable; 1: odor hardly detectable; 2 : odor slightly exceeding the threshold of malodor recognition; 3 : most certainly identifiable malodor; 4 : strong malodor; 5: very strong malodor. There are also other simpler scales where the patient speaks at different distances from the clinician who rates the odor as present or absent. ${ }^{55}$ Also, nose breath odor will be determined in order to make a differential diagnosis between intra- and extra-oral halitosis.

Other variations of the organoleptic method include collecting a mouth air sample with a syringe which the clinician then evaluates (Kim method), ${ }^{56}$ or with special bags which are specially designed to collect mouth air samples. These methods have the benefit of reducing potential bacterial cross-infection and are less embarrassing for the patient.

As mentioned above, the organoleptic method is quick, inexpensive, and does not require specialized tools but can be uncomfortable for clinicians and patients. On the other hand, before the clinician can act as a judge of oral malodor, his/her capacity to smell and recognize different odors should be tested (qualitative assessment), and also his/her capacity to detect odors at low concentrations (quantitative assessment). ${ }^{57}$ Moreover, despite the organoleptic method being recognized widely as the gold standard, some authors have noted that the organoleptic method is subjective, emotional, instinctive and indexed to the examiner's socioeconomic background and/or experiences, and lacks international calibration and standardization and may transmit microorganisms. ${ }^{58,59}$

Gas chromatography measures the concentration of VSCs separately and may be combined with mass spectrometry to enlarge the scope of the method. ${ }^{51}$ Sulfide monitors measure the total concentration of sulfides without distinction of different VSCs. Electrochemical reactions with the sulfur-containing compounds in the breath generate an electric current, which is directly proportional to sulfide levels in the patient's breath. ${ }^{51}$

Indirect diagnostic methods include Benzoyl-DLArginine-Alpha-Naphthylamide (BANA) test, ammonia monitoring, ninhydrin technique, cysteine challenge test ${ }^{60}$ plaque autofluorescence, ${ }^{61}$ and salivary incubation test, ${ }^{51}$ but the convenience, accuracy, and diagnostic value of these proposed methods has not been validated. ${ }^{59}$

\section{Treatment}

Treatment approaches for intra-oral halitosis are guided by a cause-related strategy on the basis of the diagnosis (Figure 3). The objectives of interventions for halitosis are to reduce breath odor, and thereby the individual's social functioning, with minimal adverse effects. ${ }^{33}$

Since approximately $90 \%$ of halitosis cases are of oral origin, primary halitosis management techniques should aim to reduce the bacterial biofilms present in different areas of the oral cavity, through either self-performed or professionally delivered biofilm control methods. Therefore, initial treatment approaches should include control of oral pathologies (particularly periodontal diseases) and efficient self-performed oral hygiene measures using a soft toothbrush twice a day and daily interdental cleaning including tongue cleaning measures when indicated. The tongue can be cleaned with a toothbrush, but preferably with a tongue scraper, reaching as far backward as possible since the posterior portion of the tongue harbors the most coating. Tongue cleaning should be repeated until almost no coating material can be removed. This cleaning should be gentle to prevent soft tissue damage. ${ }^{62}$ 


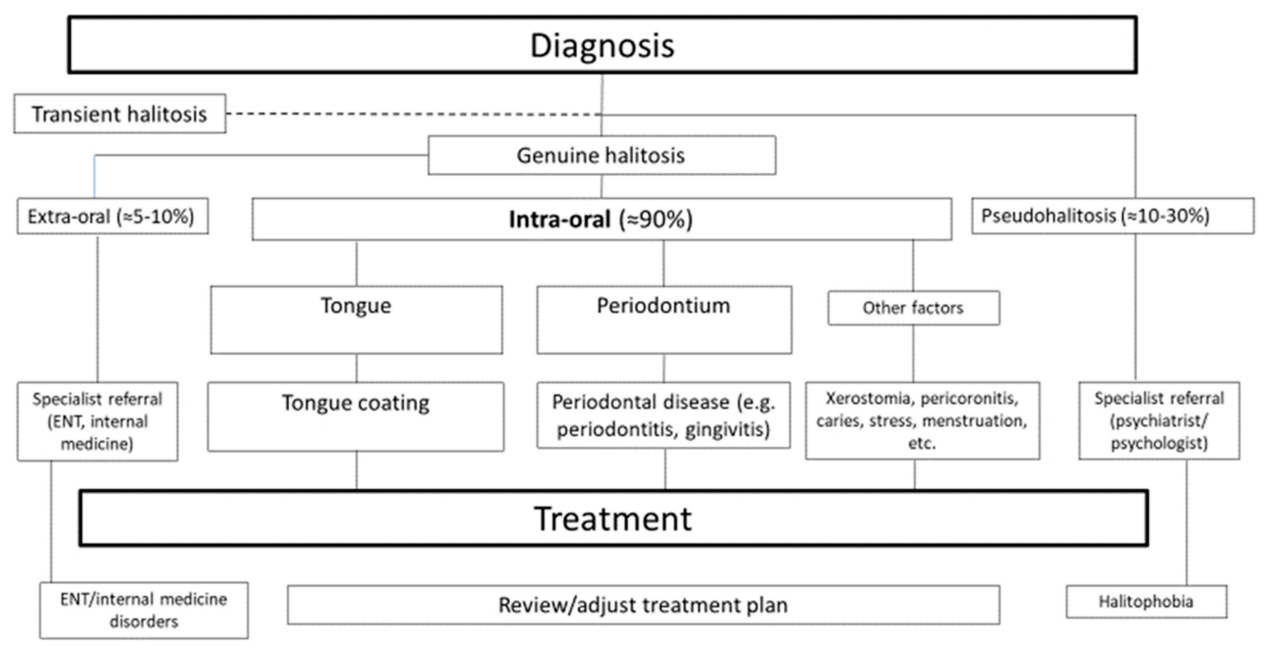

Figure 3 Schematic flowchart for the classification and diagnosis of halitosis.

Note: Adapted from Seemann R, Conceicao MD, Filippi A, et al. Halitosis management by the general dental practitioner-results of an international consensus workshop. J Breath Res. 2014;8(I):0I7I0I. Creative commons license and disclaimer available from: https://creativecommons.org/licenses/by/3.0/. ${ }^{5}$

Abbreviation: ENT, ear, nose, throat.

Tongue cleaning has been shown to reduce VSC levels by up to $75 \% .^{63}$ Seemann and co-workers compared the effectiveness of different devices in reducing oral VSC levels (a specially designed tongue cleaner [a combination of a brush and a scraper], a tongue scraper and a regular toothbrush). The tongue cleaner and the tongue scraper obtained higher VSC reductions than the toothbrush ( $42 \%, 40 \%$ and $30 \%$, respectively). This reduction lasted significantly longer after using the tongue cleaner. However, a significant VSC reduction could not be detected for more than 30 minutes in any of the subjects. ${ }^{64}$

A recent study showed that plastic or metal tongue scrapers significantly reduced WTCI scores, and anaerobic bacterial count, compared with a manual toothbrush with a tongue scraper. ${ }^{65}$ The greatest reduction in bacterial load on the dorsum of the tongue was achieved using plastic tongue scrapers. ${ }^{65}$ Another recent study has proposed a specific tongue cleaning technique, the $\mathrm{X}$ technique, for reducing the tongue biofilm and organoleptic scores in individuals with intra-oral halitosis, to improve users' perceptions of their breath. ${ }^{66}$

A Cochrane review reported a small but statistically significant reduction of VSC levels with the use of tongue scrapers or cleaners, rather than toothbrushes, to reduce halitosis in adults. ${ }^{67}$ Furthermore, a recent systematic review showed that, based on best available evidence, professional tooth cleaning, and scaling and root planing, in combination with oral hygiene instructions, reduced VSC values in patients with intra-oral halitosis and/or periodontal diseases, independently of tongue cleaning and the use of mouth rinses. ${ }^{68}$

Despite a lack of formal halitosis treatment guidelines, a simple, step-wise approach may be appropriate for the management of intra-oral halitosis (Figure 4). If intra-oral halitosis persists after treatment of oral and periodontal conditions and the implementation of efficient mechanical oral hygiene measures, an active zinc-based mouth rinse can be introduced as a coadjuvant to these mechanical biofilm control measures.

Different systematic reviews on the effect of mouth rinses on oral malodor reported that the most compelling evidence was provided for chlorhexidine (CHX) mouthwashes, and those that contained a combination of cetylpyridinium chloride and zinc, or cetylpyridinium chloride, CHX and zinc, provided the best evidence profile on oral malodor. ${ }^{69,70}$ However, the strength of evidence was graded to be "weak". ${ }^{70}$ Many commercially-available mouth rinses provide short- and long-term effects; as with most active antiseptics, CHX has some potential disadvantages when used long term, including staining of the teeth and tongue, metallic taste and reduced taste sensation. ${ }^{69}$ In addition, the alcohol content of many commercially-available mouth rinses has been associated with adverse effects, and alcohol-free mouth rinses have been shown to be effective for the treatment of intra-oral halitosis. ${ }^{71,72}$ However, there is a large body of recently published clinical evidence for therapeuticallyactive mouth rinses, particularly those containing a combination of zinc acetate and $\mathrm{CHX} .^{69,73-77}$ 


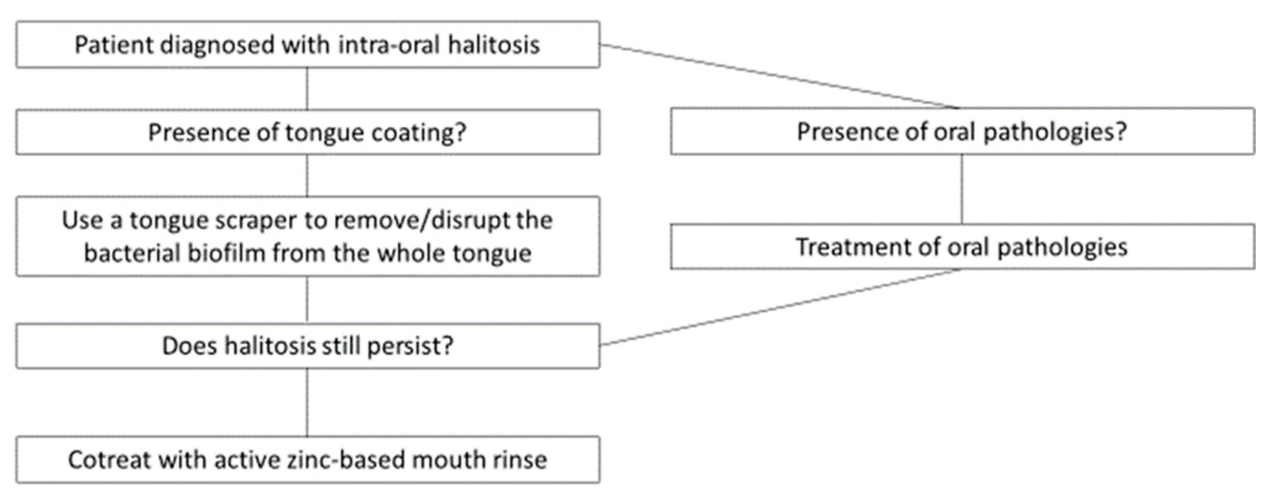

Figure 4 Simple, step-wise approach for the management of intra-oral halitosis in a general dental practice.

When comparing different treatment modalities for intraoral halitosis, the use of a tongue scraper did not provide additional benefits to an active mouth rinse (zinc acetate $0.3 \% / \mathrm{CHX} 0.025 \%$ ) but reduced organoleptic scores and the WTCI. ${ }^{73}$ In another study, an active rinse alone containing zinc acetate and CHX improved intra-oral halitosis and reduced bacterial counts of species associated with halitosis. Tongue scraping provided no beneficial effects on the microbiota studied. Thus, periodontally healthy subjects with intraoral halitosis benefit from daily rinsing with a zinc acetate/ CHX-containing mouth rinse. ${ }^{74}$

The effect of different mouth rinses $12 \mathrm{~h}$ after rinsing, was evaluated in a double-blind, crossover, randomized clinical trial (RCT) in 24 adults with intra-oral halitosis. Zinc acetate/ CHX was the most effective mouth rinse in reducing VSC levels. ${ }^{75}$ In a double-blind RCT, a mixture of zinc acetate $0.3 \%$ and CHX $0.025 \%$ showed a clear and durable effect on intraoral halitosis which lasted at least $12 \mathrm{~h}$, both during the day and overnight, with a consistent effect on both objective and subjective variables; the zinc acetate/CHX mouth rinse was well tolerated. ${ }^{76}$ Furthermore, in an RCT in 46 adults with intra-oral halitosis, zinc acetate/CHX mouth rinse provided effective long-term efficacy against intra-oral halitosis, assessed both objectively and subjectively. The zinc acetate/CHX mouth rinse provided significantly better control of intra-oral halitosis than a placebo mouth rinse. At 3 and 6 months, individuals using the zinc acetate/CHX rinse showed significant reductions in the organoleptic score and total VSCs, hydrogen sulfide and methyl mercaptan. With regular rinsing, the effect was sustained for 6 months and no differences in tooth staining were observed between study groups over time. ${ }^{77}$

Although further research is required, recent evidence from clinical studies suggests that the use of probiotics can potentially be effective for preventing and treating halitosis and periodontitis. $^{78,79}$

\section{Halitosis: Practical Considerations}

From a clinical perspective, it is crucial to distinguish between intra-oral and extra-oral halitosis. Although a small proportion of individuals have halitosis of extraoral origin and some have pseudohalitosis/halitophobia, neither of which should be underestimated, the vast majority of halitosis cases are intra-oral.

With regard to extra-oral halitosis, close collaborations with ENT or other medical specialists are needed. In these situations, the follow-up process can be difficult for patients because there is often a lack of communication/ awareness between different HCPs.

Professional multidisciplinary halitosis clinics play an important role in the management of halitosis..$^{15,16,80}$ Patients can be referred to a specialist halitosis clinic from a wide range of different HCPs, including dentists, general medical practitioners, dental hygienists, ENT specialists, gastroenterologists, pulmonary specialists, psychiatrists, and neurologists (eg patients with Parkinson's disease often lose their sense of smell in the early stages of the disease). ${ }^{81}$

With regard to the diagnosis and management of halitosis, the following section summarizes some of the main practical considerations for different HCPs and the general public.

\section{Dentists and Dental Hygienists}

In most cases, dentists and dental hygienists are ideally placed to diagnose and/or respond to an initial consultation for halitosis.

A step-wise approach is necessary for the management of intra-oral halitosis. If a patient with breath odor presents with tongue coating, the first step is to improve oral hygiene using a tongue scraper to remove/disrupt the bacterial biofilm. However, tongue cleaning/scraping alone may not always resolve the problem. If halitosis persists after improving tongue and dental hygiene and after 
treating any required periodontal or other oral pathologies, an active zinc-based mouth rinse can be introduced as a coadjuvant (never alone). In reality, a patient who perceives that they have halitosis will often want to use a mouth rinse before they have consulted with an HCP. As part of a shared decision-making process, the clinician's role should be advisory and educational, in order to ensure that patients understand that, while there is no risk to using an active mouth rinse, there are other management approaches which should ideally be evaluated before using a mouth rinse (eg tongue scraping, periodontal treatment).

In general dental practice, simple, initial diagnostic and management steps should therefore be:

(1) Patient completes a halitosis questionnaire

(2) Organoleptic testing (smell the breath) - can be done by any authorized medical practitioner with a good sense of smell (eg dentists, dental hygienists, nurses in an elderly care home, general medical practitioner)

(3) Check for the presence of plaque, periodontitis, gingivitis, and other oral pathologies

(4) Advise the patient based on (1-3), with some simple hygiene steps and possibly further periodontal examination and treatment, and treatment of other oral pathologies (see also Figure 4).

Training programs aimed at increasing the assertiveness, as well as the social and communication skills, of dentists and dental hygienists are important for improving the diagnosis and treatment of patients with halitosis. ${ }^{82}$ However, it is also important to acknowledge that diagnostic approaches differ by country (even within Europe). Oppliger and colleagues (2014) showed that there are clear differences between dentists in France and their colleagues from the German-speaking countries, but also between dental hygienists and dentists. Dental hygienists from Switzerland and Germany appear to be ahead in terms of halitosis knowledge. ${ }^{83}$ The situation in France, for example, may be compounded by the fact that there are no financial incentives for dentists to consult, diagnose and treat halitosis and this may be a demotivating factor. Another study of HCPs, undertaken in Switzerland, assessed the level of knowledge/awareness of halitosis, using a survey which was conducted over a period of 3 years in which 150 family physicians, 150 ENT specialists, 154 dentists and 151 dental hygienists were personally interviewed. ${ }^{84}$ Results showed that, despite the fact that the cause of halitosis is primarily intra-oral, patients seek first consultations with family physicians and ENT specialists.
If a patient complains of halitosis then it is easy to facilitate an open discussion about the possible cause(s) and treatment approaches. A positive approach that is employed by some clinics when consulting with periodontitis patients who have intra-oral halitosis is to ask 'Would you like to improve your breath?'. Most patients are interested in improving their breath. In order to improve the patient's breath, any periodontal diseases or other oral pathologies need to be treated, when indicated the patient needs to implement efficient self-performed oral hygiene measures with a toothbrush/interdental cleaning devices/tongue scraper and, if that does not help to improve the breath, a therapeutic mouth rinse can be introduced to their oral hygiene routines. A recent systematic review showed that, based on the best available evidence, professional tooth cleaning, and scaling and root planing, in combination with oral hygiene instructions, reduced VSC values in patients with intra-oral halitosis and/or periodontal diseases, independently of tongue cleaning and the use of mouth rinses. ${ }^{68}$ For some patients, the fact that their halitosis may not be able to be managed immediately can present a problem, so recognizing the negative impact on their quality of life and informing them of the overall management strategy can be beneficial to the patient.

\section{General Public/Patients}

It is important to raise awareness and educate the general public that the oral cavity is responsible for halitosis (approximately 90\% of cases) and that the tongue is the major source of bacteria which contribute to intra-oral halitosis. However, in general, the public are not aware of the importance of oral health, including the fact that some people should clean their tongue. ${ }^{85,86}$ Data from a representative sample of the German population (Fifth German Oral Health Study, DMS-V) ${ }^{87}$ showed that approximately $40 \%$ of patients have much plaque in their oral cavity and only $5 \%$ of the population is gingivitisfree - there is clearly room for improvement.

From the patient perspective, it is interesting to note that a study of female Saudi Arabian students $(n=392)$ found that self-perception of halitosis was low $(21.4 \%)$, but a high percentage $(78.1 \%)$ noticed other people with halitosis. Use of personal methods (eg mouthwash and chewing gum) to treat halitosis was common (77\%). Most respondents $(82.1 \%)$ considered that a dentist was the most appropriate professional to treat halitosis. ${ }^{88}$ 
Another study also reported low levels of self-perception of halitosis. ${ }^{89}$

In one study, $32 \%$ of halitosis patients reported experiencing halitosis for $>10$ years, ${ }^{16}$ indicating that many individuals are unaware of whom to contact for help. Furthermore, a survey of almost 400 young females in Saudi Arabia reported that fewer than one-quarter of participants consulted with a dentist about their perceived halitosis. ${ }^{46}$ Consequently, it is important to raise awareness among the general public that they should initially consult with their dentist/dental hygienist if they suspect the presence of halitosis.

\section{General Medical Practitioners/ Pharmacists and Other HCPs}

Many individuals will initially consult with non-dental HCPs about halitosis, but it appears that halitosis awareness and knowledge among non-dental HCPs is limited. ${ }^{90}$ Moreover, patients with pseudohalitosis are frequently not diagnosed correctly by primary care physicians, resulting in a considerable amount of costly and unnecessary overtreatment. ${ }^{14}$ Again, as the vast majority of halitosis cases are intra-oral, dentists and dental hygienists are bestplaced to diagnose and treat halitosis and there is a need for continued education/increased awareness among nondental HCPs to ensure appropriate patient referral. Giving the increased aging population, the requirement for increased awareness also applies to all personnel who handle elderly individuals in nursing homes (a large socially isolating problem among elderly care home residents). Increased exposure to halitosis awareness during the early stages of professional training, coupled with ongoing education, should assist in raising the profile of this public social health problem.

Generally, there is little connection between general practitioners (GPs) and dentists in primary care. ${ }^{91,92}$ In a qualitative study of GPs' and dentists' experiences and expectations of inter-professional collaboration in Germany, both groups of professionals reported perceived knowledge deficits regarding the other profession. ${ }^{93}$ For oral health issues, dentists expected GPs to have basic knowledge and to be able to diagnose halitosis. Indeed, whereas dentists expressed interest in extending collaboration with GPs, most GPs saw no need for collaboration. Consequently, fostering inter-professional education would be a promising way to improve long-term cooperation between GPs and dentists. $^{93}$
The concept of inter-professional collaboration to manage halitosis was also explored recently in a study of the practice, knowledge and views of Australian general dental practitioners, general medical practitioners and community pharmacists on halitosis. ${ }^{94}$ The majority of general dental practitioners, general medical practitioners and community pharmacists described that they find it challenging to manage patients with halitosis and are interested in working together. Improving health professionals' knowledge and training will contribute to comprehensive interprofessional halitosis management. ${ }^{94}$

Collaboration between HCPs would most likely improve patient outcomes. As witnessed with multidisciplinary breath odor clinics, ${ }^{15,16,80,95-97}$ different HCPs can work together to manage halitosis. Furthermore, raising community awareness is key to destigmatizing halitosis and reducing the barriers for patients to seek treatment. Educating and training HCPs by enhancing undergraduate and graduate professional curricula and promoting multidisciplinary professional development programs are critical in promoting inter-professional collaboration and providing patients with optimal care.

\section{Conclusions}

Halitosis is an underestimated problem affecting a significant proportion of the global population. Without adequate diagnosis and management, halitosis has a significant impact on quality of life and can result in psychological consequences including social, professional, and affective limitations, and in unnecessary medical procedures. The vast majority of halitosis is intra-oral and, using a simple step-wise approach for diagnosis and treatment, dentists and dental hygienists are ideally placed to respond to an initial consultation for halitosis. There is a need for increased awareness among the general public that intra-oral causes are responsible for the majority of halitosis cases. When HCPs face challenges managing patients with halitosis, increased inter-professional collaboration between HCPs (GPs, dentists, dental hygienists, community pharmacists, nurses, and medical specialists) would improve patient outcomes.

\section{Data Sharing Statement}

The data supporting the findings of this review article are available within the paper. 


\section{Acknowledgments}

Under the guidance of the authors, professional medical writing support was provided by David P. Figgitt PhD, ISMPP CMPPTM, Content Ed Net, with funding from Mylan, Spain.

\section{Author Contributions}

All authors contributed extensively to the work presented in this paper. All authors have contributed significantly to the conception and interpretation of data. All authors have participated in drafting, reviewing, and/or revising the manuscript, have approved the version to be published, and agree to be accountable for all aspects of the work.

\section{Funding}

The Advisory Board meeting, the outcomes from which form the basis for this review article, was funded by Mylan, Spain.

\section{Disclosure}

S Renvert received an honorarium from Mylan for attending the Advisory Board meeting. MJ Noack regularly acts as a scientific consultant to manufacturers of mouth rinses and home cleaning products, including CP GABA, Mylan and Johnson \& Johnson and receives honoraria for sponsored lectures; regarding the current paper, MJ Noack received an honorarium from Mylan for attending the Advisory Board meeting. C Lequart received an honorarium from Mylan for attending the Advisory Board meeting. S Roldán received personal fees for lecturing from Colgate, Dentaid and Johnson \& Johnson, not related to the present paper, and received an honorarium from Mylan for attending the Advisory Board meeting. ML Laine received a travel grant and her institute received an honorarium from Mylan for attending the Advisory Board meeting and grants from Arkray, outside the submitted work. The authors report no other conflicts of interest in this work.

\section{References}

1. American Dental Association. Halitosis. 2019. Available from https:// www.mouthhealthy.org/en/az-topics/h/halitosis. accessed 16 September 2019.

2. Kapoor U, Sharma G, Juneja M, Nagpal A. Halitosis: current concepts on etiology, diagnosis and management. Eur J Dent. 2016;10 (02):292-300. doi:10.4103/1305-7456.178294

3. UK National Health Service (NHS). Bad breath. 2019. Available from https://www.nhs.uk/conditions/bad-breath/. accessed 16 September 2019.
4. Porter SR, Scully C. Oral malodour (halitosis). BMJ. 2006;333 (7569):632-635. doi:10.1136/bmj.38954.631968.AE

5. Seemann R, Conceicao MD, Filippi A, et al. Halitosis management by the general dental practitioner-results of an international consensus workshop. J Breath Res. 2014;8(1):017101. doi:10.1088/1752$7155 / 8 / 1 / 017101$

6. Zürcher A, Laine ML, Filippi A. Diagnosis, prevalence, and treatment of halitosis. Curr Oral Health Rep. 2014;1(4):279-285. doi:10.1007/s40496-014-0036-5

7. Bicak DA. A current approach to halitosis and oral malodor- a mini review. Open Dent J. 2018;12(1):322-330. doi:10.2174/ 1874210601812010322

8. de Jongh A, van Wijk AJ, Horstman M, de Baat C. Attitudes towards individuals with halitosis: an online cross sectional survey of the Dutch general population. Br Dent J. 2014;21(4):E8. doi:10.1038/sj. bdj.2014.101

9. Eli I, Baht R, Kozlovsky A, Rosenberg M. The complaint of oral malodor: possible psychopathological aspects. Psychosom Med. 1996;58(2):156-159. doi:10.1097/00006842-199603000-00010

10. Conceicao MDD, Giudice FS, Carvalho LF. The Halitosis Consequences Inventory: psychometric properties and relationship with social anxiety disorder. BDJ Open. 2018;4(1):18002. doi:10.1038/bdjopen.2018.2

11. Lu HX, Chen XL, Wong M, Zhu C, Ye W. Oral health impact of halitosis in Chinese adults. Int J Dent Hyg. 2017;15(4):e85-e92. doi:10.1111/idh.12242

12. Zaitsu T, Ueno M, Shinada K, Wright FA, Kawaguchi Y. Social anxiety disorder in genuine halitosis patients. Health Qual Life Outcomes. 2011;9(1):94. doi:10.1186/1477-7525-9-94

13. Patel J, Kulkarni A, Doshi D, Reddy P, Reddy S, Srilatha A. Association between social anxiety with oral hygiene status and tongue coating among patients with subjective halitosis. J Dent Hyg. 2017;91(5):55-63.

14. Seemann R, Bizhang M, Djamchidi C, Kage A, Nachnani S. The proportion of pseudo-halitosis patients in a multidisciplinary breath malodour consultation. Int Dent J. 2006;56(2):77-81. doi:10.1111/ j.1875-595X.2006.tb00077.x

15. Quirynen M, Dadamio J, Van den Velde S, et al. Characteristics of 2000 patients who visited a halitosis clinic. J Clin Periodontol. 2009;36(11):970-975. doi:10.1111/j.1600-051X.2009.01478.x

16. Zürcher A, Filippi A. Findings, diagnoses and results of a halitosis clinic over a seven year period. Schweiz Monatsschr Zahnmed. 2012;122(3):205-216.

17. De Geest S, Laleman I, Teughels W, Dekeyser C, Quirynen M. Periodontal diseases as a source of halitosis: a review of the evidence and treatment approaches for dentists and dental hygienists. Periodontol 2000. 2016;71(1):213-227. doi:10.1111/prd.12111

18. Silva MF, Cademartori MG, Leite FRM, López R, Demarco FF, Nascimento GG. Is periodontitis associated with halitosis? A systematic review and meta-regression analysis. $J$ Clin Periodontol. 2017;44(10):1003-1009. doi:10.1111/jcpe.12786

19. Porter SR, Scully C, Hegarty AM. An update of the etiology and management of xerostomia. Oral Surg Oral Med Oral Pathol Oral Radiol Endod. 2004;97(1):28-46. doi:10.1016/j. tripleo.2003.07.010

20. Koshimune S, Awano S, Gohara K, Kurihara E, Ansai T, Takehara T. Low salivary flow and volatile sulfur compounds in mouth air. Oral Surg Oral Med Oral Pathol Oral Radiol Endod. 2003;96(1):38-41. doi:10.1016/S1079-2104(03)00162-8

21. Nalcaci R, Baran I. Oral malodor and removable complete dentures in the elderly. Oral Surg Oral Med Oral Pathol Oral Radiol Endod. 2008;105(6):e5-9. doi:10.1016/j.tripleo.2008.02.016

22. Van Tornout M, Dadamio J, Coucke W, Quirynen M. Tongue coating: related factors. J Clin Periodontol. 2013;40(2):180-185. doi:10.1111/ jcpe. 12031 
23. Lu HX, Tang C, Chen X, Wong MC, Ye W. Characteristics of patients complaining of halitosis and factors associated with halitosis. Oral Dis. 2014;20(8):787-795. doi:10.1111/odi.12198

24. Chen X, Zhang Y, Lu HX, Feng XP. Factors associated with halitosis in white-collar employees in Shanghai, China. PLoS One. 2016;11 (5):e0155592. doi:10.1371/journal.pone.0155592

25. Seerangaiyan K, Jüch F, Winkel EG. Tongue coating: its characteristics and role in intra-oral halitosis and general health-a review. J Breath Res. 2018;12(3):034001. doi:10.1088/17527163/aaa3a1

26. Seerangaiyan K, Maruthamuthu M, van Winkelhoff AJ, Winkel EG. Untargeted metabolomics of the bacterial tongue coating of intra-oral halitosis patients. J Breath Res. 2019;13(4):046010. doi:10.1088/ $1752-7163 / \mathrm{ab} 334 \mathrm{e}$

27. Koga C, Yoneda M, Nakayama K, et al. The detection of candida species in patients with halitosis. International Journal of Dentistry. 2014;2014:857647. doi:10.1155/2014/857647

28. Aydin M, Derici MÇ, Ünal Y, Yeler D, Demir Yİ. Is there any relation between type 1 halitosis and oral candida colonisation? Mikrobiyol Bul. 2019;53(2):192-203. doi:10.5578/mb.67759

29. Tangerman A, Winkel EG. Extra-oral halitosis: an overview. J Breath Res. 2010;4(1):017003. doi:10.1088/1752-7155/4/1/017003

30. Ferguson M, Aydin M, Mickel J. Halitosis and the tonsils: a review of management. Otolaryngol Head Neck Surg. 2014;151(4):567-574. doi:10.1177/0194599814544881

31. Torsten M, Gómez-Moreno G, Aguilar-Salvatierra A. Drug-related oral malodour (halitosis): a literature review. Eur Rev Med Pharmacol Sci. 2017;21(21):4930-4934.

32. Aydin M, Harvey-Woodworth CN. Halitosis: a new definition and classification. $\quad B r \quad$ Dent $J . \quad 2014 ; 217(1): E 1 . \quad$ doi:10.1038/sj. bdj.2014.552

33. Scully C. Halitosis. BMJ Clin Evid. 2014;2014:1305.

34. Silva MF, Leite FRM, Ferreira LB, et al. Estimated prevalence of halitosis: a systematic review and meta-regression analysis. Clin Oral Investig. 2018;22(1):47-55. doi:10.1007/s00784-017-2164-5

35. Rösing CK, Loesche W. Halitosis: an overview of epidemiology, etiology and clinical management. Braz Oral Res. 2011;25 (5):466-471. doi:10.1590/S1806-83242011000500015

36. Nazir MA, Almas K, Majeed MI. The prevalence of halitosis (oral malodor) and associated factors among dental students and interns, Lahore, Pakistan. Eur J Dent. 2017;11(04):480-485. doi:10.4103/ejd. ejd_142_17

37. Ueno $\bar{M}$, Ohnuki M, Zaitsu T, Takehara S, Furukawa S, Kawaguchi Y. Prevalence and risk factors of halitosis in Japanese school children. Pediatr Int. 2018;60(6):588-592. doi:10.1111/ ped. 13561

38. Du M, Li L, Jiang H, Zheng Y, Zhang J. Prevalence and relevant factors of halitosis in Chinese subjects: a clinical research. BMC Oral Health. 2019;19(1):45. doi:10.1186/s12903-019-0734-4

39. Madhushankari GS, Yamunadevi A, Selvamani M, Mohan Kumar KP, Basandi PS. Halitosis - an overview: part-I - classification, etiology, and pathophysiology of halitosis. J Pharm Bioallied Sci. 2015;7(6):S339-S343. doi:10.4103/0975-7406.163441

40. Zellmer M, Gahnberg L, Ramberg P. Prevalence of halitosis in elderly living in nursing homes. Int $J$ Dent Hyg. 2016;14 (4):295-300. doi:10.1111/idh.12236

41. Guedes CC, Bussadori SK, Weber R, et al. Halitosis: prevalence and association with oral etiological factors in children and adolescents. J Breath Res. 2019;13(2):026002. doi:10.1088/17527163/aafc6f

42. Villa A, Zollanvari A, Alterovitz G, Cagetti MG, Strohmenger L, Abati S. Prevalence of halitosis in children considering oral hygiene, gender and age. Int J Dent Hyg. 2014;12(3):208-212. doi:10.1111/idh.12077

43. Lin MI, Flaitz CM, Moretti AJ, Seybold SV, Chen JW. Evaluation of halitosis in children and mothers. Pediatr Dent. 2003;25(6):553-558.
44. Schmidt J, Krause F, Haak R. Halitosis: measurement in daily practice. Quintessence Int. 2015;46(7):633-641. doi:10.3290/j.qi.a34134

45. Hammad AM, Darwazeh AM, Al-Waeli H, Tarakji B, Alhadithy TT. Prevalence and awareness of halitosis in a sample of Jordanian population. J Int Soc Prev Community Dent. 2014;4(6):S178-S186. doi:10.4103/2231-0762.149033

46. Ali SAA, Sadatullah S, Al-Deleh HF, AlDossari LJ, Alqahtani SD, Alshahrani BA. The prevalence of self-perceived halitosis among a sample of young Saudi females residing in Abha - kingdom of Saudi Arabia. KKU J Health Sci. 2017;2:18-24.

47. Miyazaki H, Sakao S, Katoh Y, Takehara T. Correlation between volatile sulphur compounds and certain oral health measurements in the general population. $J$ Periodontol. 1995;66(8):679-684. doi:10.1902/jop.1995.66.8.679

48. Winkel EG, Roldán S, Van Winkelhoff AJ, Herrera D, Sanz M. Clinical effects of a new mouthrinse containing chlorhexidine, cetylpyridinium chloride and zinc-lactate on oral halitosis. a dual-center, double-blind placebo-controlled study. J Clin Periodontol. 2003;30 (4):300-306. doi:10.1034/j.1600-051X.2003.00342.x

49. Roldán S, Herrera D, Santa-Cruz I, O’Connor A, González I, Sanz M. Comparative effects of different chlorhexidine mouth-rinse formulations on volatile sulphur compounds and salivary bacterial counts. J Clin Periodontol. 2004;31(12):1128-1134. doi:10.1111/ j.1600-051X.2004.00621.X

50. Lundgren T, Mobilia A, Hallström H, Egelberg J. Evaluation of tongue coating indices. Oral Dis. 2007;13(2):177-180. doi:10.1111/ j.1601-0825.2006.01261.x

51. van den Broek AM, Feenstra L, de Baat C. A review of the current literature on aetiology and measurement methods of halitosis. J Dent. 2007;35(8):627-635. doi:10.1016/j.jdent.2007.04.009

52. Hatt H. Molecular and cellular basis of human olfaction. Chem Biodivers. 2004:1:1857-1869.

53. Rosenberg M, McCulloch CA. Measurement of oral malodor: current methods and future prospects. J Periodontol. 1992;63(9):776-782. doi:10.1902/jop.1992.63.9.776

54. Greenman J, Lenton P, Seemann R, Nachnani S. Organoleptic assessment of halitosis for dental professionals - general recommendations. J Breath Res. 2014;8(1):017102. doi:10.1088/1752-7155/8/1/017102

55. Bornstein MM, Kislig K, Hoti BB, Seemann R, Lussi A. Prevalence of halitosis in the population of the city of Bern, Switzerland: a study comparing self-reported and clinical data. Eur J Oral Sci. 2009;117 (3):261-267. doi:10.1111/j.1600-0722.2009.00630.x

56. Kim DJ, Lee JY, Kho HS, Chung JW, Park HK, Kim YK. A new organoleptic testing method for evaluating halitosis. J Periodontol. 2009;80(1):93-97. doi:10.1902/jop.2009.080389

57. Doty RL, Shaman P, Dann M. Development of the University of Pennsylvania Smell Identification Test: a standardized microencapsulated test of olfactory function. Physiol Behav. 1984;32:489-502. doi:10.1016/0031-9384(84)90269-5

58. Quirynen M, Zhao H, Avontroodt P, et al. A salivary incubation test for evaluation of oral malodor: a pilot study. J Periodontol. 2003;74 (7):937-944. doi:10.1902/jop.2003.74.7.937

59. Aydin M, Bollen CM, Özen ME. Diagnostic value of halitosis examination methods. Compend Contin Educ Dent. 2016;37(3):174-178.

60. Kleinberg I, Codipilly DM. Cysteine challenge testing: a powerful tool for examining oral malodour processes and treatments in vivo. Int Dent J. 2002;52(Suppl 3):221-228. doi:10.1002/j.1875595X.2002.tb00929.X

61. Lee ES, Yim HK, Lee HS, Choi JH, Kwon HK, Kim BI. Plaque autofluorescence as potential diagnostic targets for oral malodor. J Biomed Opt. 2016;21(8):85005. doi:10.1117/1.JBO.21.8.085005

62. Clark GT, Nachnani S, Messadi DV. Detecting and treating oral and nonoral malodors. J Calif Dent Assoc. 1997;25(2):133-144.

63. Tonzetich J. Oral malodour: an indicator of health status and oral cleanliness. Int Dent J. 1978;28(3):309-319. 
64. Seemann R, Kison A, Bizhang M, Zimmer S. Effectiveness of mechanical tongue cleaning on oral levels of volatile sulfur compounds. J Am Dent Assoc. 2001;132(9):1263-1267. doi:10.14219/jada.archive.2001.0369

65. Dwivedi V, Torwane NA, Tyagi S, Maran S. Effectiveness of various tongue cleaning aids in the reduction of tongue coating and bacterial load: a comparative clinical study. J Contemp Dent Pract. 2019;20 (4):444-448. doi:10.5005/jp-journals-10024-2536

66. Gonçalves ACS, Martins MCM, Paula BL, Weckwerth PH, Franzolin SOB, Silveira EMV. A new technique for tongue brushing and halitosis reduction: the X technique. J Appl Oral Sci. 2019;27: e20180331. doi:10.1590/1678-7757-2018-0331

67. Outhouse TL, Al-Alawi R, Fedorowicz Z, Keenan JV. Tongue scraping for treating halitosis. Cochrane Database Syst Rev. 2006;2: CD005519.

68. Deutscher H, Derman S, Barbe AG, Seemann R, Noack MJ. The effect of professional tooth cleaning or non-surgical periodontal therapy on oral halitosis in patients with periodontal diseases. A systematic review. Int $J$ Dent Hyg. 2018;16(1):36-47. doi:10.1111/idh.12306

69. Blom T, Slot DE, Quirynen M, Van der Weijden GA. The effect of mouthrinses on oral malodor: a systematic review. Int J Dent Hyg 2012;10(3):209-222. doi:10.1111/j.1601-5037.2012.00546.x

70. Slot DE, De Geest S, van der Weijden FA, Quirynen M. Treatment of oral malodour. Medium-term efficacy of mechanical and/or chemical agents: a systematic review. J Clin Periodontol. 2015;42:S303-S316. doi:10.1111/jcpe. 12378

71. Gagari E, Kabani S. Adverse effects of mouthwash use. A review. Oral Surg Oral Med Oral Pathol Oral Radiol Endod. 1995;80 (4):432-439. doi:10.1016/S1079-2104(05)80337-3

72. Aydin M, MÇ D, Keskek SO, Demir YI, Yeler D. Instant and freshness effect of mouth rinses on type 1 (oral) halitosis. Acta Odontol Latinoam. 2019;32(2):79-87.

73. Erovic Ademovski S, Lingström P, Winkel E, Tangerman A, Persson GR, Renvert S. Comparison of different treatment modalities for oral halitosis. Acta Odontol Scand. 2012;70(3):224-233. doi:10.3109/00016357.2011.635601

74. Ademovski SE, Persson GR, Winkel E, Tangerman A, Lingström P, Renvert S. The short-term treatment effects on the microbiota at the dorsum of the tongue in intra-oral halitosis patients-a randomized clinical trial. Clin Oral Investig. 2013;17(2):463-473. doi:10.1007/ s00784-012-0728-y

75. Erovic Ademovski S, Lingström P, Renvert S. The effect of different mouth rinse products on intra-oral halitosis. Int J Dent Hyg. 2016;14 (2):117-123. doi:10.1111/idh.12148

76. Seemann R, Filippi A, Michaelis S, Lauterbach S, John HD, Huismann J. Duration of effect of the mouthwash CB12 for the treatment of intra-oral halitosis: a double-blind, randomised, controlled trial. J Breath Res. 2016;10(3):036002. doi:10.1088/17527155/10/3/036002

77. Erovic Ademovski S, Mårtensson C, Persson GR, Renvert S. The long-term effect of a zinc acetate and chlorhexidine diacetate containing mouth rinse on intra-oral halitosis-a randomized clinical trial. J Clin Periodontol. 2017;44(10):1010-1019. doi:10.1111/jcpe.12779

78. Georgiou AC, Laine ML, Deng DM, Brandt BW, van Loveren C, Dereka X. Efficacy of probiotics: clinical and microbial parameters of halitosis. J Breath Res. 2018;12(4):046010. doi:10.1088/1752-7163/ aacf49

79. Bustamante M, Oomah BD, Mosi-Roa Y, Rubilar M, Burgos-Díaz C. Probiotics as an adjunct therapy for the treatment of halitosis, dental caries and periodontitis. Probiotics Antimicrob Proteins. 2019. doi:10.1007/s12602-019-9521-4
80. Schumacher M, Zürcher A, Filippi A. Evaluation of a halitosis clinic over a period of eleven years. Swiss Dent J. 2017;127:846-851.

81. Barbe AG, Deutscher DHC, Derman SHM, Hellmich M, Noack MJ. Subjective and objective halitosis among patients with Parkinson's disease. Gerodontology. 2017;34(4):460-468. doi:10.1111/ger.12293

82. Buunk-Werkhoven YA, Buls JG, Osinga E, Bruers JJ. Diagnosis and treatment of patients with halitosis by dental hygienists and dentists in the Netherlands. Int Dent J. 2015;65(2):65-70. doi:10.1111/ idj. 12145

83. Oppliger N, Roth B, Filippi A. Knowledge of halitosis among dentists and dental hygienists. Swiss Dent J. 2014;124(2):133-143.

84. Roth B, Oppliger N, Filippi A. Knowledge of different medical and dental professional groups in Switzerland about halitosis. Swiss Dent J. 2014;124:1302-1312.

85. Kapoor D, Gill S, Singh A, Kaur I, Kapoor P. Oral hygiene awareness and practice amongst patients visiting the Department of Periodontology at a Dental College and Hospital in North India. Indian J Dent. 2014;5:64-68. doi:10.4103/0975-962X.135262

86. Singh A, Gambhir RS, Singh S, Kapoor V, Singh J. Oral health: how much do you know? - A study on knowledge, attitude and practices of patients visiting a North Indian dental school. Eur J Dent. 2014;8:63-67. doi:10.4103/1305-7456.126244

87. Jordan RA, Bodechtel C, Hertrampf K, et al. The Fifth German Oral Health Study (Fünfte Deutsche Mundgesundheitsstudie, DMS V) rationale, design, and methods. BMC Oral Health. 2014;14:161. doi:10.1186/1472-6831-14-161

88. Bin Mubayrik A, Al Hamdan R, Al Hadlaq EM, et al. Selfperception, knowledge, and awareness of halitosis among female university students. Clin Cosmet Investig Dent. 2017;9:45-52. doi:10.2147/CCIDE.S129679

89. Humagain M, Dixit S, Bhandari B, Khanal S, Singh PK. Selfperception of halitosis among undergraduate students of kathmandu university school of medical sciences - a questionnaire based study. Kathmandu Univ Med J (KUMJ). 2018;16(61):89-93.

90. Afolabi AO, Ogundipe OK, Eleckwachi G. Halitosis (bad breath): an assessment of the awareness and knowledge of health providers in a Nigerian community. Sudan Med J. 2009;45:42-48.

91. Holzinger F, Dahlendorf L, Heintze C. 'Parallel universes'? The interface between GPs and dentists in primary care: a qualitative study. Fam Pract. 2016;33(5):557-561. doi:10.1093/fampra/cmw058

92. Huettig F, Said FM, Sippli K, Preiser C, Rieger MA. What do general practitioners and dentists report about their cooperation? A qualitative exploration [Article in German]. Gesundheitswesen. 2018;80:262-265.

93. Sippli K, Rieger MA, Huettig F. GPs' and dentists' experiences and expectations of interprofessional collaboration: findings from a qualitative study in Germany. BMC Health Serv Res. 2017;17 (1):179. doi:10.1186/s12913-017-2116-4

94. Lau P, Meethal C, Middleton M, Clark M, Darby I. 'Say Ahhh': what do dentists, general medical practitioners and community pharmacists do about halitosis? International Dental Journal. 2009;117 (4):311-320. doi:10.1111/idj.12458

95. Delanghe G, Ghyselen J, van Steenberghe D, Feenstra L. Multidisciplinary breath-odour clinic. Lancet. 1997;350(9072):187. doi:10.1016/S0140-6736(05)62354-9

96. Delanghe G, Ghyselen J, Feenstra L, van Steenberghe D. Experiences of a Belgian multidisciplinary breath odour clinic. Acta Otorhinolaryngol Belg. 1997;51:43-48.

97. Ben-Aryeh H, Horowitz G, Nir D, Laufer D. Halitosis: an interdisciplinary approach. Am J Otolaryngol. 1998;19:8-11. doi:10.1016/ S0196-0709(98)90058-8 


\section{Publish your work in this journal}

Clinical, Cosmetic and Investigational Dentistry is an international, peer-reviewed, open access, online journal focusing on the latest clinical and experimental research in dentistry with specific emphasis on cosmetic interventions. Innovative developments in dental materials, techniques and devices that improve outcomes and patient satisfaction and preference will be highlighted. The manuscript management system is completely online and includes a very quick and fair peer-review system, which is all easy to use. Visit http://www.dovepress.com/testimonials.php to read real quotes from published authors.

Submit your manuscript here: https://www.dovepress.com/clinical-cosmetic-and-investigational-dentistry-journal 